\title{
ÉTICA Y MODELO DE LOS PRINCIPIOS
}

\author{
Ma Teresa López de la Vieja
}

Universidad de Salamanca

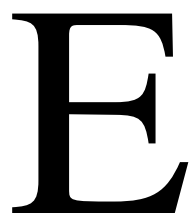

n un artículo de 1998, R. Posner denunciaba la falta de respuestas prácticas que ofrecen la Teoría moral y algunos filósofos, a quienes se refería como «moralistas académicos». R. Posner criticaba con dureza las limitaciones de la Teoría moral contemporánea, por ser una base sumamente deficiente para guiar los juicios morales, o para orientar las decisiones en el ámbito jurídico. En este alegato contra el «moralismo académico» ${ }^{1}$, Posner mencionaba expresamente a autores contemporáneos, tan diferentes entre sí como R. Dworkin, J. Rawls, J. Raz, Th. Nagel, M. Nussbaum. Todos ellos representan -en su opinión- un tipo de teoría que ha perdido definitivamente su creatividad. Los especialistas ya no son innovadores, no están a la altura de las demandas de los agentes, su trabajo no sirve para mejorar la conducta, ni siquiera resuelven sus propios desacuerdos teóricos... R. Posner pintaba una situación general muy desfavorable, en la cual los debates que realmente importan tenían lugar fuera del ámbito académico, era mejor explicar los fenómenos morales sin el concurso de las categorías morales, etc. El artículo retomaba los consabidos argumentos contra los excesos de la teoría, contra la separación entre teoría y vida práctica, entre categorías morales y dilemas prácticos, la incapacidad de los especialistas para hablar de lo que realmente motiva a los agentes. A cambio, defendía una versión de la moralidad ligada a lo contextual y a las intuiciones, dedicada a explicar las cuestiones prácticas, a cambiar las conductas, e incluso a ofrecer un nuevo punto de vista.

No es la primera vez que la Filosofía moral contemporánea ha sido objeto de críticas de este tipo, contra los excesos del análisis teórico y contra su falta de relevancia. Esta actitud negativa hacia lo teórico pretende devolver la primacía a lo práctico, tampoco es nueva aunque se presente bajo fór-

\footnotetext{
${ }^{1}$ Posner, R.: «The Problematics of Moral and Legal Theory», Harvard Law Review, 111, 1998, pp. 1638-1709.
} 
mulas radicales. En los años ochenta, S. Toulmin abogaba ya por una nueva casuística, denunciando la rigidez o «tiranía» ${ }^{2}$ de los principios de carácter absoluto. De este modo pretendía que las teorías se mantuvieran fieles a la complejidad de los problemas prácticos, resolviendo los casos particulares de forma prudente, discreta, sin la ayuda de reglas ni de principios. Sin embargo, la Filosofía moral contemporánea no ha logrado una posición de consenso sobre el tema, escindida todavía entre las cuestiones de principio y las formas de vida particulares, entre partidarios de un modelo normativo fuerte y, de otro lado, partidarios de aligerar la teoría, a fin de no perder el contacto con la experiencia moral. Por tanto, sería un error tomar al pie de la letra las objeciones de R. Posner y de S. Toulmin, como si fuesen «despedidas» de la teoría. En conjunto, los temas morales relevantes plantean casi siempre cuestiones de Metaética, al tiempo que las teorías normativas se ocupan de un modo u otro -directo o indirecto- de cuestiones prácticas $^{3}$. Ficticias son también las tensiones fuertes entre «fundamentación» y «aplicación», o entre Teoría ética y éticas aplicadas. Es decir, un genuino interés por los aspectos teóricos y prácticos terminará por reducir las distancias entres los «moralistas académicos» -en los términos de R. Posner-y los agentes que deliberan sobre cuestiones morales.

R. Posner llamaba la atención sobre el valor del sentido común y de la experiencia moral. Sin embargo, exageraba los errores y las deficiencias de vocabulario y de metodología que lastran al «moralismo académico». En todo caso, la hostilidad hacia las teorías no es un método alternativo, ni la falta de relevancia se subsana apelando tan sólo al sentido común como criterio. Digamos que la Metaética es bastante vulnerable a las críticas, pero éstas no llegan muy lejos sin un análisis especializado, cuidadoso en el uso del vocabulario moral. Según este mismo criterio, el modelo de principios será tan vulnerable como parece serlo el «moralismo académico» $\mathrm{y}$, sin embargo, resulta difícil analizar las cuestiones prácticas que realmente preocupan al agente sin el concurso de los principios morales. En las páginas siguientes se va a defender este punto de vista, rechazando, por tanto, las objeciones fuertes contra la Teoría moral: (1) los principios morales, sobre todo los principios prima facie, son lo suficientemente flexibles como para responder a las demandas de aplicabilidad, sin debilitar el aparato teórico y crítico de la Filosofía. (2) Hay que construir teorías relevantes, es cierto. Teorías que expliquen lo que sucede en la experiencia, en los casos reales, etc. Pero las teorías tienen siempre una aplicabilidad restringida, al no ofrecer un catálogo de soluciones para cada caso. (3) El mejor ajuste entre princi-

\footnotetext{
${ }^{2}$ Toulmin, S.: «The Tyranny of Principles», The Hastings Center Report, 6, 1981, pp. 31-39.

${ }^{3}$ Me he interesado por el equilibrio entre casos y principios en el volumen Principios morales y casos prácticos, Tecnos, Madrid, 2000, pp. 17-35.
} 
pios y casos prácticos nunca podrá sustituir a la iniciativa y a la decisión de los mismos agentes, de la misma forma que la Teoría ética no ha sido reemplazada por éticas especiales, de pretensiones excesivas y con un aparato conceptual reducido. En suma, las teorías normativas y los principios prima facie son interesantes para aquellos agentes que tienen distintas visiones del mundo, teniendo que deliberar sobre problemas prácticos similares. Por eso mismo necesitan normas flexibles, así como teorías para analizar los casos, argumentos bien definidos, principios prima facie, etc.

El artículo recuerda el papel central que pueden desempeñar tanto el modelo de los principios, entendidos éstos como «mandatos óptimos»-según la terminología de R. Alexy-, como la aplicabilidad restringida en el análisis de las cuestiones morales. La idea de una «teorización incompleta» -como la denomina C. Sunstein- restaría fuerza a las críticas de R. Posner, y de todos aquellos autores que han hecho una excesiva valoración del sentido común. La renuncia a la teoría, el menosprecio de una tradición de debates, pulida por la controversia, por el análisis, etc., simplifican las cosas sólo en apariencia. En una situación como la actual, de pluralismo y simultaneidad de puntos de vista, los agentes no necesitan realmente soluciones para cada caso, sino condiciones favorables para deliberar y para actuar con autonomía. Y algunos principios para «dibujar la línea» de lo correcto, lo justo, lo tolerable, etc.

\section{El modelo de los principios}

Las Eticas cognitivistas son limitadas, tienen un contenido muy reducido, no consideran los motivos reales de los agentes, tienden al universalismo irrestricto, etc. Todas las críticas aciertan en su diagnóstico general. Sin embargo, la Casuística tampoco carece de dificultades a la hora de aplicar su método a las circunstancias particulares, encontrándose además bajo el peso de tradiciones ${ }^{4}$ que hoy no podríamos asumir sin reservas. Por un lado, la Filosofía moral contemporánea ha derivado algunas veces hacia debates puramente teóricos, poco relevantes; por otro lado, las éticas especiales o «aplicadas» han abierto expectativas que no podían cumplir sin el apoyo de un fuerte aparato teórico. Por tanto, el rechazo hacia la gran teoría moral suele estar acertada en algunos casos, pero en otros casos ofrece soluciones débiles o insuficientes. Sin duda, el modelo de los principios carece del atractivo que rodea a las éticas que comienzan con andanadas contra la teoría, también suscitan menos interés que las éticas «aplicadas».

En cambio, este modelo aporta consistencia a la discusión sobre cuestiones prácticas de una cierta complejidad o envergadura. Los principios

${ }^{4}$ Para el método del caso y sus ventajas sobre las reglas y los principios, Jonsen, A., Toulmin, S.: The Abuse of Casuistry, University of California Press, Berkeley, 1988, pp. 1-20. 
funcionan, por así decirlo, como la base que posibilitará un acuerdo entre agentes que mantienen distintos puntos de vista. En un contexto abierto, como ocurre en las sociedades pluralistas, este choque de formas de ver y formas de vivir es lo habitual; los principios nunca impiden tales desacuerdos, ni borran por sí solos las distancias. Y, sin embargo, aportan cierto equilibrio, algo así como un punto de referencia para cualquier discusión complicada. Son la expresión más sintética de los compromisos básicos que los agentes podrían asumir, sin tener que renunciar a sus propios puntos de vista: los principios marcan una línea clara que nadie debería rebasar, sean cuáles sean las circunstancias particulares. «Dibujan la línea» según la expresión de R. Nozick ${ }^{5}$.

(a) Los principios son un tipo de normas, prescriben algo que tiene que hacerse, indican una conducta a adoptar. La dificultad ${ }^{6}$ para definirlos de manera precisa tiene que ver con las dificultades que plantea el mismo lenguaje moral, por su estructura abierta ${ }^{7}$. Normas y reglas se dicen de muchas maneras, he ahí la primera consecuencia. Por ejemplo, el término «regla» se refiere a algo que se debe o que se ha de hacer, si bien hablamos también de acciones racionales en el caso de acciones que estén gobernadas por reglas. Según la definición de R. Alexy, los principios son «mandatos óptimos» ${ }^{8}$, puesto que prescriben algo a realizar, pero a realizar de forma gradual, en mayor o en menor medida. En cambio, las reglas funcionan de otra forma, pues se trata de «mandatos definitivos», ya que sólo cabe cumplirlas o no cumplirlas, sí o no, sin gradación alguna. Esto significa que los principios se realizan a niveles distintos; como «mandatos óptimos». Entonces permitirán también una relación abierta con los casos prácticos. Prescriben algo, pero sin determinar el modo, el momento ni el grado mismo de la realización. Y esto resulta sumamente interesante para reforzar el papel de las normas en la discusión práctica ${ }^{9}$. Por tanto, los principios crean un marco bien definido, pero flexible. Además existen varios principios, de manera que habrá conflicto entre éstos, por eso hará falta deliberar y sopesar las razones para preferir un «mandato óptimo» a otro mandato. Esto significa, en suma, que no existen principios absolutos, sino principios que valen mientras la

${ }^{5}$ Nozick, R.: The Nature of Rationality, Princeton University Press, Princeton, 1993, pp. 3-40.

${ }^{6}$ Señalada en su momento por G.H. Von Wright, Norma y acción, Tecnos, Madrid, 1979, pp. 21-35.

${ }^{7}$ Para la estructura abierta de este tipo de lenguaje, Brennan, J.M.: The Open-Texture of Moral Concepts, MacMillan, London, 1977, pp. 88-149.

${ }^{8}$ Alexy, R.: Recht, Vernunft, Diskurs, Suhrkamp, Frankfurt, 1995, pp. 237-238; Theorie der Grundrechte, Nomos, Baden-Baden, 1985, pp. 71-157.

${ }_{9}^{9}$ Para el lugar de los principios en la argumentación jurídica, Atienza, M.: Tras la justicia, Ariel, Barcelona, 1993, pp.27-28. 
información disponible no demuestre lo contrario. Esta despedida de los principios absolutos resulta sumamente útil en aquellos casos prácticos más conflictivos como, por ejemplo, la interrupción del embarazo, que tanto sufrimiento y polémica ha generado. ¿Hubiera sido todo más sencillo sin el peso de principios tan fuertes como la santidad de la vida?

(b) Los principios prima facie no son principios absolutos. W.D. Ross ${ }^{10}$ se refería a los «deberes condicionados» o deberes de obligación imperfecta: prescriben algo, pero sólo en el caso de que no haya otro deber más significativo. Por esta razón, parecen relevantes las circunstancias propias de la situación, de tal manera que los principios son necesarios, pero no autosuficientes. Los principios prima facie introducen un enfoque especialmente interesante para las teorías cognitivistas, ya que permiten sostener que la argumentación práctica es racional, que hay analogía entre los criterios de verdad y la corrección práctica ${ }^{11}$. Y, al mismo tiempo, estos principios reducen las pretensiones sobre los casos y sobre los contenidos concretos. Por tanto, este modelo puede introducir cierto equilibrio entre las expectativas desmesuradas, que han abierto las éticas especiales, y la aplicabilidad indirecta de Teoría moral, tan criticada desde el sentido común.

Se puede decir que los principios establecen las condiciones para analizar los casos prácticos a partir de criterios racionales. Sólo las condiciones, un marco flexible, una aplicabilidad mediada por otros factores, etc. Pues las decisiones sobre el caso conciernen a los individuos o a los grupos; los principios sólo garantizan un punto de partida, algo así como cierta estabilidad en las razones para la acción. En general, hablamos de no matar, de no torturar, no privar de libertad, etc.; los agentes dirán cuál ha de ser la solución más correcta para cada situación. Los principios, entendidos como «razones para la acción» ${ }^{12}$, son un punto de partida, cuya fuerza deriva de que no haya otras razones de tipo superior. En este sentido, se dice que los principios «dibujan líneas» y sirven para justificar las decisiones. Pero «justificar» no es lo mismo que «aplicar»o tomar decisiones.

\section{Aplicabilidad restringida}

J. Narveson ${ }^{13}$ ya había llamado la atención sobre el estatuto impreciso de los principios. En este mismo sentido, D. Emmet ${ }^{14}$ se refería a los dos

\footnotetext{
${ }^{10}$ Ross, W. D.: The Right and the Good, Clarendon, Oxford, 1967, pp. 16-47

${ }^{11}$ Habermas,J.: «On the Cognitive Content of Morality», Proceedings of the Aristotelian Society, 1996, pp. 335-358

${ }_{12}$ Raz, J.: Razón práctica y normas, Centro de Estudios Constitucionales, Madrid, 1991, pp. $55-96$.

${ }^{13}$ Narveson, J.: Morality and Utility, J.Hopkins University Press, Baltimore, 1967, pp. 21-50.

${ }^{14}$ Emmet, D.: Rules, Roles and Relations, St.Martin's Press, New York, 1966, pp. 56-88.
} 
usos de las «reglas morales», uso regulativo, como directrices para una conducta, y uso constitutivo, guía de actos específicos. Las cuestiones de terminología han sido consideradas también J. Searle ${ }^{15}$, quien reconoce finalmente la ausencia de explicaciones claras sobre el concepto de «prima facie». Por su parte, R. Dworkin ${ }^{16}$ distingue entre «principios» -no definen un programa a seguir- $\mathrm{y}$, de otro lado, una «directiz» (policy) que define un programa de actuación. Por lo tanto, el modelo de los principios es complejo y presenta dificultades terminológicas. Ninguna de estas dificultades elimina, sin embargo, la función codificadora de las normas, la capacidad para sistematizar que tienen los principios ${ }^{17}$

(1) Según Nozick, la función codificadora interviene en tres niveles, nivel intelectual, interpersonal y personal. La función intelectual consiste en aportar justificaciones, guiar las acciones y las decisiones, para que puedan ser consideradas como decisiones correctas. La función interpersonal introduce cierta seguridad en la relación de los agentes, ya que los principios definen un tipo de interacción relativamente independiente o al margen de intereses circunstanciales, aumentando así la confianza en las relaciones personales. La función personal consiste en definir la propia identidad, ya que los principios pueden actuar a modo de filtro, aportando estabilidad o definiendo un comportamiento a largo plazo, hacia el futuro. Por tanto, el rendimiento de los principios es doble, ya que establecen un marco general para la acción y, además, ofrecen una información relevante para la deliberación práctica. R. Nozick afirma que los principios «dibujan la línea», en el sentido de que sistematizan y aportan racionalidad.

(2) Los principios pueden funcionar también como premisas o como punto de partida para la argumentación práctica. Sin este punto de partida, será imposible sopesar, poner en la balanza las razones a favor y las razones en contra de una decisión concreta. Por un lado, se puede decir que los casos constituyen un auténtico test para demostrar la aplicabilidad de los principios, de otro lado, los principios introducen una forma específica de ver los casos, y de hallar soluciones adecuadas para cada situación. Es más, el punto de partida, el modo según el cual se define una situación, determinará en buena medida el tipo de respuesta o la conclusión del razonamiento sobre los casos prácticos. Por ejemplo, la Etica medioambiental -tanto en su forma más radical, biocéntrica, como en su forma más moderada o antropocéntrica- apela al principio de preservación, para favorecer un trato más respetuoso con las otras especies. Sin embargo, la estrategia y los re-

${ }^{15}$ Searle, J.: «Obligaciones prima facie », en: Raz,J.: Razonamiento práctico, FCE, Mexico, 1986, pp. 153-170.

${ }^{16}$ Dworkin, R.: A Matter of Principle, Harvard University Press, London, 1985, pp. 1-6.

${ }_{17}$ Nozick, R.: The Nature of Rationality, pp.3-40. 
sultados concretos serán distintos, según sea el principio al que se apele. Al introducir el principio de justicia, se dirá que los agentes han de favorecer el trato equitativo entre generaciones y entre especies. Si, por el contrario, lo decisivo fuese el valor intrínseco de las especies, se dirá que los agentes han de evitar daños a los seres vivos, como seres valiosos por sí mismos, más allá de una consideración instrumental.

A lo largo de los últimos años, el interés por los casos prácticos ha propiciado el crecimiento de las éticas especiales, como disciplinas con un desarrollo independiente -como sucede con la Etica medioambiental-, e incluso con un desarrollo opuesto al de la Filosofía moral contemporánea. Algunos autores, como S. Toulmin ${ }^{18}$, han llegado a pensar que la Medicina había «salvado la vida» a la Etica. Otros, como A. MacIntyre ${ }^{19}$ ha llegado a la conclusión opuesta, que no hay una «nueva» Ética, sino bastantes malentendidos sobre los resultados de las disciplinas especiales. Por tanto, el rechazo de la teoría y la excesiva valoración de las éticas aplicadas podría derivar de una apreciación equivocada de lo que significa «aplicabilidad», cuando no la consolidación de intereses profesionale ${ }^{20}$ restringidos. En definitiva, no resulta plausible una división del trabajo entre Etica general y éticas aplicadas ${ }^{21}$; es más, el papel central de la teoría destaca más cuanto más se desarrollan estas éticas especiales. Pues las cuestiones prácticas exigen claridad y precisión teórica. Desde una perspectiva externa, el «hecho del pluralismo» ${ }^{22}$ tampoco conduce a una proliferación de éticas especiales, de códigos de conducta, de soluciones para el caso, etc., sino que muestra las ventajas de una Etica con pretensiones reducidas, en beneficio de agentes competentes y con autonomía para tomar decisiones.

\section{Normas flexibles}

Las críticas sobre el carácter abstracto de la Ética y de los principios aciertan sólo en parte. Ni los principios pueden dejar de ser abstractos ${ }^{23}$

${ }_{18}$ Toulmin, S.: «How Medicine Saved the Life of Ethics», en: De Marco, J.P., Fox, R.M., New Directions in Ethics, Routledge a. Kegan Paul, London, 1986, pp. 265-281.

${ }_{19}$ MacIntyre, A.:«Does Applied Ethics Rest On a Mistake?», The Monist , 1984, pp. 498-513.

${ }^{20} \mathrm{El}$ papel de los intereses corporativos y profesionales en el desarrollo de las éticas especiales ha sido comentado por V.Held, Rights and Goods. Justifiying Social Action, University of Chicago Press, 1984, pp. 1-7.

${ }_{21}$ Sobre los problemas derivados de una división del trabajo en este campo, Brown, J.: «On Applying Ethics», en: Evans, J.D., Moral Philosophy and Contemporary Problems, Cambridge University Press, Cambridge, 1987, pp.81-93.

${ }_{22}$ Rawls, J.: Political Liberalism, Columbia University Press, New York, 1993, pp. XIIIXXXIV.

${ }^{23}$ La idea de que los principios han de ser necesariamente abstractos se encuentra en O’Neill, O.: «Abstraction, Idealization and Ideology in Ethics», en: Evans, J.D., Moral Philosophy and Contemporary Problems, pp. 55-69. 
-como razones para la acción ${ }^{24}$ - ni tiene sentido buscar un algoritmo para los casos prácticos. Los principios prima facie muestran distintos grados de obligación y, por tanto, favorecen el ajuste con los casos. Esto es, el argumento de R. Posner contra el «moralista académico» resultó ser menos demoledor de lo que aparentaba. En cambio, las observaciones de C. Sunstein sobre la función positiva de los desacuerdos en las sociedades heterogéneas pueden debilitar el papel central de las reglas en los argumentos prácticos. Por un motivo análogo al que se acaba de mencionar, algunas veces importa más lo que aún queda por decir, las circunstancias cambiantes y las necesidades del individuo que los acuerdos y las teorías completas.

1. El hecho de que se produzcan habitualmente serios desacuerdos sobre los valores fundamentales puede ser una buena señal. Una señal de apertura, de sensibilidad hacia lo particular, actitudes tolerantes, respeto por formas de vida y por los juicios morales, etc. Está claro que el énfasis sobre lo particular no es compatible con reglas demasiado rígidas, pero tampoco con reglas demasiado generales, que fuesen inaplicables o irrelevantes. En este contexto de rechazo de teorías y de principios de alto nivel, C. Sunstein propone que empecemos a «teorizar de forma incompleta» ${ }^{25}$. Justamente para no renunciar a reglas y principios, haciéndolos flexibles pero no ciegos hacia lo particular. Este autor admite tanto las ventajas de situaciones donde ya existen reglas claras, imparciales -pues minimizan los costes en el proceso de toma de decisiones, reducen la incertidumbre en los casos particulares, evitan situaciones desagradables a los ciudadanos, al definir el marco de sus derechos, permiten planificar, identificar responsabilidades, etc.-, como a las ventajas de la analogía entre casos similares. C. Sunstein defiende la adaptación de las reglas a los casos, para reducir los posibles riesgos, derivados tanto de la ausencia de reglas como de lo contrario, reglas ciegas e impersonales.

2. La mayoría de las teorías normativas han estado buscando algo parecido, una estrategia para ajustar las necesidades reales y las normas imparciales. Por tanto, se interesan por un modelo tan alejado de los principios dogmáticos como de los casos atípicos. ¿Puede haber un procedimiento lo suficientemente amplio como para recoger ambos elementos? El concepto de «aplicabilidad reducida» y los principios prima facie forman parte de una idea de reflexión moral, entendida como equilibrio y ajuste entre lo

${ }^{24}$ Aarnio, A., Peczenik, A.: «On Values. Universal or Relative?», Ratio Juris, 1996, pp. 321-330

${ }^{25}$ Sunstein, C.: Legal Reasoning and Political Conflict, Oxford University Press, New York, 1996, pp. 3-12. 
universal y lo particular. También sirven para consolidar ${ }^{26}$ una la Teoría moral postradicional, pero no postmoderna. Los argumentos de D. Ross, R. Dworkin, R. Nozick, C. Sunstein ayudan a explicar por qué optar por un modelo de principios desde el momento en que las sociedades valoran tanto la cooperación como las preferencias individuales. Por ejemplo, O. Höf$\mathrm{fe}^{27}$ considera a los principios como el contrapunto necesario de la cultura moderna, plural. En definitiva, los principios serán tan sólo un punto de partida; la complejidad de las demandas y de los intercambios revaloriza cualquier punto de partida que esté bien definido. Se trata de trazar líneas básicas, los agentes dirán luego si quieren y cómo quieren realizar el equilibrio entre los principios, entre principios y casos reales.

A modo de conclusión, puede decirse que los principios establecen un nivel óptimo o un ideal ${ }^{28}$, cuya validez se encuentra al margen de intereses o de enfoques particulares. En ese sentido, los principios morales definen o «dibujan la línea» de lo aceptable y de lo inaceptable. Su fuerza y aplicabilidad dependerán de los agentes, por eso los principios sólo tienen la fuerza de las razones, y una aplicabilidad reducida o indirecta. Los principios, como «mandatos óptimos», traducen a normas lo que dice el punto de vista moral, ese punto de vista que está por encima - pero no al margen- de formas de vida y del contexto. Por tanto, los principios no son absolutos ni ignoran las contingencias o la situación real de los agentes. Son algo distinto, la expresión de lo normativo, así como un instrumento para dar razón de las decisiones, para deliberar sobre los casos prácticos. En el mismo sentido en que G. Ryle ${ }^{29}$ decía que conocer una regla es, también, saber usar una regla.

De un lado, las sociedades pluralistas y heterogéneas potencian la decisión individual, debilitando la fuerza de los valores y de los principios absolutos. Por eso mismo, carece ya de sentido la figura del filósofo como moralista, alguien que se arroga la facultad de hablar en nombre de los demás agentes. Agentes que, por lo demás, son perfectamente competentes para elegir su forma de vida, su sistema de valores, e incluso para encontrar por sí mismos la solución correcta para los problemas prácticos. De otro lado, estas mismas sociedades requieren de ciertos valores, así como de ciertas prácticas que favorezcan la cooperación y la deliberación entre los ciudadanos. Los principios morales, como hemos visto, no dicen qué prácticas, qué

${ }^{26}$ B. Gert apuesta tanto por una Teoría moral desarrollada -para organizar las intuiciones morales- como por la Etica aplicada, «Moral Theory and Applied Ethics», The Monist, 67, 1984, pp. 532-548.

${ }^{27}$ Höffe, O.: Kategorische Rechtsprinzipien, Suhrkamp, Frankfurt, 1995, pp. 11-29.

${ }_{28}$ Peczenik, A.: On Law and Reason, Kluwer, Dordrecht, 1989, pp. 47-117.

${ }^{29}$ Ryle, G.: «Knowing How and Knowing That», Proceedings of the Aristotelian Society, XLVI, 1945-46, pp. 1-16. 
valores, qué conductas habrán que adoptar en cada situación. Tan sólo establecen las premisas de la discusión, algo así como un marco general a fin de encontrar las mejores razones para la acción. Las críticas de R. Posner contra la Filosofía normativa señalaban algunos de los muchos defectos que percibimos en el trabajo de los filósofos. Sin embargo, los argumentos ad hominen no demostraban que los principios morales y la teoría sean innecesarios; son, tal vez, un acicate para mostrar la relevancia ${ }^{30}$ de la Filosofía moral.

${ }^{30}$ Ante las quejas expresadas por R. Posner en su artículo, R. Dworkin abogaba por la indispensabilidad de la Teoría moral, «Darwin's New Bulldog», Harvard Law Review, 11, 1998, pp. 1718- 1738 .

\section{DOXA 23 (2000)}

